Anais da Academia Brasileira de Ciências (2017) 89(4): 2563-2579

(Annals of the Brazilian Academy of Sciences)

Printed version ISSN 0001-3765 / Online version ISSN 1678-2690

http://dx.doi.org/10.1590/0001-3765201720170233

www.scielo.br/aabc | www.fb.com/aabcjournal

\title{
Periodic complex map germs and foliations
}

\author{
LEONARDO M. CÂMARA and BRUNO A. SCÁRDUA
}

\author{
Universidade Federal do Espírito Santo, Departamento de Matemática - CCE, \\ Av. Fernando Ferrari, 514, 29075-910 Vitória, ES, Brazil \\ Universidade Federal do Rio de Janeiro, Instituto de Matemática, Caixa Postal 68530, \\ 21945-970 Rio de Janeiro, RJ, Brazil
}

Manuscript received on April 10, 2017; accepted for publication on June 1, 2017

\begin{abstract}
In this paper we study topological and analytical conditions on the orbits of a germ of diffeomorphism in the complex plane in order to obtain periodicity. In particular, we give a simple proof of a finiteness criteria for groups of analytic diffeomorphisms, stated in Brochero Martínez 2003. As an application, we derive some consequences about the integrability of complex vector fields in dimension three in a neighborhood of a singular point.
\end{abstract}

Key words: Complex germs of diffeomorphims, singular holomorphic foliations, integrability of vector fields, closed orbits.

\section{1 - INTRODUCTION}

The relationship between periodic subgroups of $\operatorname{Diff}(\mathbb{C}, 0)$ and the integrability of germs of vector fields at $\left(\mathbb{C}^{2}, 0\right)$ was established in Mattei and Moussu 1980. There the authors show that the topological condition of finiteness of the orbits is sufficient to ensure the periodicity of a finitely generated sugbroup at $\operatorname{Diff}(\mathbb{C}, 0)$. As a consequence, they obtain that the topological condition of closeness of the orbits of a germ of vector field $X$ is equivalent to the existence of a first integral, i.e., a germ of map $f:\left(\mathbb{C}^{2}, 0\right) \longrightarrow(\mathbb{C}, 0)$ whose level sets contain the orbits of $X$. The link between these two objects is obtained in terms of the so called holonomy group, introduced by Charles Ehresmann.

In this paper we show that for $n=2$ a completely distinct phenomena occur. In fact we present and explicit example (Example 2.1) showing that the finiteness of the orbits of a cyclic subgroup of $\operatorname{Diff}\left(\mathbb{C}^{2}, 0\right)$ is not enough to ensure its periodicity. From this example we construct a vector field $X$ at $\left(\mathbb{C}^{3}, 0\right)$ (Example 3.1) whose leaves are closed but are not contained in the level sets of a map-germ $F:\left(\mathbb{C}^{3}, 0\right) \longrightarrow\left(\mathbb{C}^{2}, 0\right)$.

In the sequel we show that the periodicity of a germ of diffeomorphism at $\left(\mathbb{C}^{2}, 0\right)$ is achieved if we add one further topological condition, i.e., the finiteness of the orbits together with the Lyapunov stability

AMS Classification: Primary 37F75, 57R30; Secondary 32M25, 32S65.

Correspondence to: Leonardo Meireles Câmara

E-mail: leonardo.camara@ufes.br 
condition. In terms of vector fields this may be translated by saying that $X$ admits a first integral $F$ : $\left(\mathbb{C}^{3}, 0\right) \longrightarrow\left(\mathbb{C}^{2}, 0\right)$ if and only if its orbits are closed off the origin and transversely stable (in the sense of Lyapunov and Reeb) with respect to a distinguished smooth separatrix.

In the final part of the paper we show that these new topological aspects may be reinterpreted in terms of flags of foliations (Theorem 3.4). Finally we want to mention that we also present in this paper a simple proof of a finiteness criteria for groups of analytic diffeomorphisms (Theorem 2.2), stated in Brochero Martínez 2003 , since it is one of our main ingredients.

\section{2 - GERMS OF DIFFEOMORPHISMS WITH FINITE ORBITS}

\section{1 - PRELIMINARIES}

Let $\operatorname{Diff}\left(\mathbb{C}^{n}, 0\right)$ denote the group of germs of diffeomorphisms of $\mathbb{C}^{n}$ fixing the origin. Let $G \in \operatorname{Diff}\left(\mathbb{C}^{2}, 0\right)$ and $V$ be a neighborhood of the origin, where a representative (also denoted by $G$ ) of the germ $G$ is defined. Then we denote by

$$
\mathcal{O}_{V}^{+}(G, x)=\left\{G^{\circ(n)}(x): G^{\circ(j)}(x) \in V, j=0, \ldots, n\right\}
$$

the so-called positive semiorbit of $x \in V$ by $G$. Analogously, the negative semiorbit of $x \in V$ by $G$ is the set $\mathcal{O}_{V}^{-}(G, x):=\mathcal{O}_{V}^{+}\left(G^{-1}, x\right)$. The orbit of $x \in V$ by $G$ is the set $\mathcal{O}_{V}(G, x)=\mathcal{O}_{V}^{+}(G, x) \cup \mathcal{O}_{V}^{-}(G, x)$. The cardinality of $\mathcal{O}_{V}(G, x)$ is denoted by $\left|\mathcal{O}_{V}(G, x)\right|$.

A diffeomorphism $f \in \operatorname{Diff}\left(\mathbb{C}^{n}, 0\right)$ is said to be tangent to the identity if it can be expanded in series of homogeneous polynomials as $f=\left(f_{1}, \ldots, f_{n}\right)$ with $f_{j}(z)=z_{j}+f_{j, \nu_{j}}(z)+\cdots$, where $f_{j, \nu_{j}} \not \equiv 0$ and $\operatorname{ord}\left(f_{j, k}\right)=k$ whenever $f_{j, k} \not \equiv 0$. Then one says that $\nu(f)=\min \left\{\nu_{1}, \ldots, \nu_{n}\right\}$ is the order of $f$. The set of germs of diffeomorphisms tangent to the identity is denoted by $\operatorname{Diff}_{1}\left(\mathbb{C}^{n}, 0\right)$.

Let $f \in \operatorname{Diff}_{1}\left(\mathbb{C}^{2}, 0\right)$, then we say that $\varphi: \Omega \longrightarrow \mathbb{C}^{n}$ is a parabolic curve for $f$ at the origin if it is an injective holomorphic map satisfying the following properties:

(1) $\Omega \subset \mathbb{C}$ is a simply connected domain with $0 \in \partial \Omega$;

(2) $\varphi$ is continuous at the origin and $\varphi(0)=0 \in \mathbb{C}^{n}$;

(3) $\varphi(\Omega)$ is invariant under $f$ and $\left(\left.f\right|_{\varphi(\Omega)}\right)^{\circ(n)} \rightarrow 0 \in \mathbb{C}^{n}$ as $n \rightarrow \infty$.

Let $f(x)=x+f_{\nu}(x)+O\left(\|x\|^{\nu+1}\right)$, where $f_{\nu}(x)=\left(f_{1, \nu}(x), f_{2, \nu}(x)\right) \not \equiv 0$ with $f_{j, \nu}$ being homogeneous polynomial of degree $\nu$. Then we say that $f$ is dicritical if $x_{2} f_{1, \nu}(x)-x_{1} f_{2, \nu}(x) \equiv 0$ and non-dicritical otherwise. The relationship between parabolic curves and dicritical fixed points is given by the following result.

Theorem 2.1 (Abate 2001). Let $f \in \operatorname{Diff}_{1}\left(\mathbb{C}^{2}, 0\right)$ be a dicritical germ of holomorphic map tangent to the identity, then $f$ admits infinitely many parabolic curves.

\section{2 - INFINITELY MANY INVARIANT SETS}

In Brochero Martínez 2003 the following result is announced as Theorem 3.1, but unfortunately its proof seems to be incomplete. 
Theorem 2.2. Let $G \in \operatorname{Diff}\left(\mathbb{C}^{2}, 0\right)$, then the group generated by $G$ is finite if and only if there exists a neighborhood $V$ of the origin such that $\left|\mathcal{O}_{V}(G, x)\right|<\infty$ for all $x \in V$ and $G$ preserves infinitely many analytic invariant curves at 0 .

Here we shall present a simple and consistent proof for it. Since the necessary part of Theorem 2.2 is trivial, then we only prove its sufficient part in the following series of claims. For this sake, we need to recall some terminology from complex dynamics.

A germ of curve $S \subset\left(\mathbb{C}^{2}, 0\right)$ is a separatrix for $f$ if $f(S)=S$ as germs of curves at the origin. Such a separatrix is called periodic of period $n \in \mathbb{N}$ if $f^{\circ(n)}(x)=x$ for each $x \in S$. The curve $S$ is not necessarily irreducible and may have several branches. The map $f$ takes a branch into a branch and may interchange these branches. Nevertheless, since $S$ has only a finite number of branches, we conclude that for each branch $S_{j} \subset S$ there is $1 \leq m_{j} \in \mathbb{N}$ such that $f^{\circ\left(m_{j}\right)}\left(S_{j}\right)=S_{j}$. Then clearly we have $f^{\circ(m)}\left(S_{j}\right)=S_{j}$, for all $j=1, \ldots, r$, where $m=m_{1} \cdots m_{r}$. Thus, we have:

Claim 1. For each separatrix $S$ of $f$ there is $m_{S} \in \mathbb{N}$ such that each branch $S_{j}$ of $S$ is invariant with respect to $f^{\circ\left(m_{S}\right)}$.

Remark 2.1. It is well-known that a map germ $h \in \operatorname{Diff}(\mathbb{C}, 0)$ with finite orbits is necessarily periodic (Mattei and Moussu 1980). Let now $S \subset\left(\mathbb{C}^{2}, 0\right)$ be an irreducible separatrix of $f \in \operatorname{Diff}\left(\mathbb{C}^{2}, 0\right)$. Put $h=\left.f\right|_{S}$. We claim that if $h$ has finite orbits then $h$ is periodic. Indeed, in case $S$ is (irreducible and) smooth this is immediate. In the general (irreducible) case we take a Newton-Puiseux parametrization $\sigma:(\mathbb{C}, 0) \rightarrow$ $(S, 0)$. This is a holomorphic injective map so that we may consider the "lift" $\bar{h}:(\mathbb{C}, 0) \rightarrow(\mathbb{C}, 0)$, i.e., the holomorphic map that satisfies $\sigma \circ \bar{h}=h \circ \sigma$. Then $\bar{h} \in \operatorname{Diff}(\mathbb{C}, 0)$ also has finite orbits and thus, is periodic. Therefore, the same holds for $h$.

Combining Claim 1 and Remark 2.1 we promptly obtain:

Claim 2. For any separatrix $S$ of $f \in \operatorname{Diff}\left(\mathbb{C}^{2}, 0\right)$ there exists $N(S) \in \mathbb{N}$ such that $\left.f\right|_{S}$ is periodic of period $N(S)$, i.e., $\left(\left.f\right|_{S}\right)^{\circ(N(S))}=\left.\left(f^{\circ(N(S))}\right)\right|_{S}=$ Id.

In view of the above result, from now on we suppose that $f$ admits infinitely many periodic separatrices.

We say that a set of separatrices for $f$ is in general position if their first tangent cone intersect the exceptional divisor in at least three distinct points.

Claim 3. Let $f \in \operatorname{Diff}\left(\mathbb{C}^{2}, 0\right)$ and $\left\{S_{j}\right\}_{j=1}^{\infty}$ be distinct periodic separatrices for $f$, then after a finite number of blowing-ups on $S$ there appears a local map-germ $\widetilde{f} \in \operatorname{Diff}\left(\mathbb{C}^{2}, 0\right)$ admitting an infinite set of irreducible periodic separatrices in general position.

Proof. It is immediate that after blowing-up $S$ a finite number of times at least three distinct curves will intersect the exceptional divisor in three distinct points in such a way that one of these points intersects infinitely many separatrices.

Claim 4. Suppose $f \in \operatorname{Diff}\left(\mathbb{C}^{2}, 0\right)$ has a set of irreducible periodic separatrices in general position. Then there exists $n \in \mathbb{N}$ such that $f^{\circ(n)}$ is tangent to the identity.

Proof. Let $S_{j}, j=1,2,3$, be three distinct periodic separatrices in general position of orders respectively $n_{j}$. Let $n=n_{1} n_{2} n_{3}$, then $g:=f^{\circ n}$ has three distinct irreducible separatrices in general position. Now, let 


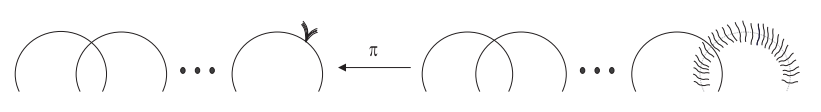

Figure 1 - Blowing-up infinitely many separatrices.

$\widetilde{g}$ be the strict transform of $g$ by a blow-up $\pi: \mathcal{M} \longrightarrow D$. If $D=\pi^{-1}(0)$ is the exceptional divisor of this blow-up, then it follows immediately from the hypothesis that $\left.\widetilde{g}\right|_{D}=$ Id. Let us prove that the first jet of $g$ is given by a diagonal matrix. Let $g=\left(g_{1}, g_{2}\right)$, where $J^{1} g_{j}(x, y)=\alpha_{j} x+\beta_{j} y$. After one blowing-up we have

$$
\widetilde{g}(t, x)=\left(\frac{g_{2}(x, t x)}{g_{1}(x, t x)}, g_{1}(x, t x)\right)=\left(\frac{\alpha_{2}+\beta_{2} t}{\alpha_{1}+\beta_{1} t}+x(\cdots), g_{1}(x, t x)\right) .
$$

Since $\left.\widetilde{g}\right|_{D}=\mathrm{Id}$, then $\alpha_{2}=\beta_{1}=0$ and $\alpha:=\alpha_{1}=\beta_{2} \in \mathbb{C}^{*}$. Therefore, $J^{1} g(x, y)=(\alpha x, \alpha y)$. Now, suppose the three irreducible invariant curves are smooth (or resolved after one blowing-up), then we may suppose without loss of generality that $S:(y=0)$ is an invariant periodic curve for $g$. In particular, its strict transform $\widetilde{S}:(t=0)$ is periodic with respect to $\widetilde{g}$. Since $\left.\widetilde{g}\right|_{D}(0, x)=\left(0, g_{1}(x, 0)\right)=(0, \alpha x+\cdots)$, then $\alpha=\exp \left(\frac{2 n \pi i}{m}\right)$ is a root of unity. The result then follows. On the other hand, suppose $S$ is a singular irreducible curve invariant by $g$ (not necessarily periodic) and recall Remark 2.1. Let $\sigma(s)=\left(s^{m}, \mu s^{n}+\cdots\right)$ be the Newton-Puiseux parametrization for $\widetilde{S}$ and $\bar{g} \in(\mathbb{C}, 0)$ be given by $\sigma \circ \bar{g}(s)=\widetilde{g} \circ \sigma(s)$. If $\bar{g}(s)=$ $\lambda s+\cdots$, then

$$
\left(\lambda^{m} s^{m}+\cdots, \mu \lambda^{n} s^{n}+\cdots\right)=\widetilde{g}\left(s^{m}, \mu s^{n}+\cdots\right)=\left(s^{m}+\cdots, \alpha \mu s^{n}+\cdots\right),
$$

thus $\lambda^{m}=1$ and $\alpha=\lambda^{n}$; i.e., $\alpha=\exp \left(\frac{2 k n \pi i}{m}\right)$ is a root of unity. The result then follows.

Claim 5. Suppose $g \in \operatorname{Diff}_{1}\left(\mathbb{C}^{2}, 0\right)$ admits an infinite number of separatrices, then along its resolution $\pi:(\mathcal{M}, D) \longrightarrow\left(\mathbb{C}^{2}, 0\right)$ there appears a dicritical map-germ $\widetilde{g} \in(\mathcal{M}, p)$ tangent to the identity in a neighborhood of $p \in D$.

Proof. Let $\widetilde{g}$ be the strict transform of $g$ by a resolution $\pi: M \longrightarrow\left(\mathbb{C}^{2}, 0\right)$, then to each invariant curve $S$ there corresponds a fixed point for $\widetilde{g}$ along $\mathcal{M}$. Therefore, at least one of the projective spaces composing the exceptional divisor $D=\pi^{-1}(0)$ admits infinitely many separatrices for $g$ transversal to it. The result then follows.

Therefore, in order to prove Theorem 2.2 we just have to combine the above claims with Abate's theorem.

\section{3 - INVARIANT FUNCTIONS}

A finitely generated subgroup $G \subset \operatorname{Diff}(\mathbb{C}, 0)$ is finite provided that it has finite pseudo-orbits (Mattei and Moussu 1980). Contrasting with the one dimensional case, in greater dimensions the finiteness of the orbits is not enough to ensure the periodicity of the group.

Example 2.1. Consider the map $G(x, y)=\left(x+y^{2}, y\right)$. The orbits of $G$ are confined in the level sets of $f(x, y)=y$ and are clearly finite. Notice that $\# \mathcal{O}_{V}(G,(x, y)) \rightarrow \infty$ as $y \rightarrow 0$, thus $G$ is not periodic nor linearizable. Furthermore, the orbits $\mathcal{O}_{V}(G,(x, y))$ are far from being stable, since in each line $(y=c)$ the map $G$ acts as a translation. 
Blowing up this diffeomorphism (cf. Abate 2001) $G=\left(g_{1}, g_{2}\right)=\left(x+y^{2}, y\right)$ at the origin one has

$$
\begin{aligned}
\widetilde{G}(t, x) & =\left(\frac{g_{2}(x, t x)}{g_{1}(x, t x)}, g_{1}(x, t x)\right)=\left(\frac{t}{1+t^{2} x}, x+t^{2} x^{2}\right) \\
& =\left(t\left(1-t^{2} x^{2}+t^{4} x^{4}-t^{6} x^{6}+\cdots\right), x\left(1+t^{2} x\right)\right) \\
& =\left(t-t^{3} x^{2}+t^{5} x^{4}-t^{7} x^{6}+\cdots, x+t^{2} x^{2}\right)
\end{aligned}
$$

whose orbits are finite and confined in the level sets of $\tilde{f}(t, x)=t x$. Further, $G$ acts in these level sets of $\tilde{f}$ in some sort of translation whose orbits increase in cardinality as $\tilde{f}(t, x) \rightarrow 0$.

Therefore in order to obtain periodicity we need to ask some further conditions. We say that two germs of holomorphic functions $f, g \in \mathcal{O}_{2}$ are generically transverse if $d f \wedge d g$ is not identically zero.

Theorem 2.3. Let $f, g \in \mathcal{O}_{2}$ be generically transverse germs and $G \in \operatorname{Diff}\left(\mathbb{C}^{2}, 0\right)$ be a complex map germ having finite orbits and preserving the level sets of both $f$ and $g$. Then $G$ is periodic.

Proof. The idea of the proof is the following: Since $f$ and $g$ are generically transverse, then one can find a pure meromorphic function $h_{o}=f_{o} / g_{o}$ whose level sets are preserved by $G$. Hence, the infinitely many curves $f_{o}(x, y)-c \cdot g_{o}(x, y)=0$, with $c \in \mathbb{C}$, intersect the origin and are invariant by $G$. Thus Theorem 2.2 ensures that $G$ is periodic. Now let us construct $h_{o}$. If $f / g$ is already pure meromorphic, then it is enough to pick $h_{o}:=f / g$. Otherwise one has $f=h \cdot g^{k}$, where $k \in \mathbb{Z}_{+}$, and $h$ is a germ of holomorphic function not divisible by $g$. Clearly, $h$ is $G$-invariant, thus if it has an irreducible component distinct from the irreducible components of $g$, then $h / g$ must be a $G$-invariant pure meromorphic function. Suppose that the decomposition in irreducible components of $g$ and $h$ are of the form $g=g_{1}^{p_{1}} \cdots g_{n}^{p_{n}}$ and $h=g_{1}^{q_{1}} \cdots g_{n}^{q_{n}}$. Since $h$ is not divisible by $g$, then there must be $j_{0} \in\{1, \cdots, n\}$ such that $q_{j_{0}}<p_{j_{0}}$. If there is also $j_{1} \in\{1, \cdots, n\}$ such that $q_{j_{1}}>p_{j_{1}}$, then $h / g$ is a pure meromorphic $G$-invariant function. From now on we suppose that $q_{j} \leq p_{j}$ for all $j=1, \ldots, n$ with at least one $j_{0} \in\{1, \cdots, n\}$ such that $q_{j_{0}}<p_{j_{0}}$. If there is $j_{1} \in\{1, \cdots, n\}$ such that $q_{j_{1}}=p_{j_{1}}$, then after reordering the indexes (if necessary) we may suppose that: (i) $q_{i}<p_{i}$ for all $i=1, \ldots, n_{0}$; (ii) $q_{i}=p_{i}$ for all $i=n_{0}+1, \cdots, n$; for some $n_{0} \in$ $\{1, \cdots, n-1\}$. Then $\bar{h}:=g / h=g_{1}^{p_{1}-q_{1}} \cdots g_{n_{0}}^{p_{n_{0}}-q_{n_{0}}}$ is a $G$-invariant germ of holomorphic function. Now, let $s_{1}:=\left[p_{1} /\left(p_{1}-q_{1}\right)\right]+1$ (where $[x]$ denotes the integer part of $\left.x \in \mathbb{R}\right)$, then a straightforward calculation shows that $g / h^{s_{1}}$ is a pure meromorphic function. Hereafter we suppose that $q_{j}<p_{j}$ for all $j=1, \ldots, n$. Recall that the Euclid's algorithm of a pair of positive integers $(p, q), p>q$, is the sequence of pairs of positive integers $\left\{\left(p_{j}, q_{j}\right)\right\}_{j=1}^{n+1}$ given by: (1) $\left(p_{1}, q_{1}\right):=(p, q)$; (2) $p_{j}=q_{j} \cdot r_{j}+s_{j}$, where $r_{j}:=[p / q]$ and $s_{j}<q_{j}$; (3) $\left(p_{j+1}, q_{j+1}\right):=\left(q_{j}, r_{j}\right)$; and (4) $s_{n}>0$ and $s_{n+1}=0$. This is called the Euclid's sequence of the pair $(p, q)$. For simplicity, suppose that $g$ and $h$ have only two irreducible components, say $g=f^{p}(\bar{f})^{\bar{p}}$ and $h=f^{q}(\bar{f})^{\bar{q}}$, and let $\left\{\left(p_{j}, q_{j}\right)\right\}_{j=1}^{n+1}$ and $\left\{\left(\bar{p}_{j}, \bar{q}_{j}\right)\right\}_{j=1}^{n+1}$ be the Euclid's sequence of $(p, q)$ and $(\bar{p}, \bar{q})$, respectively. If $r_{1}=\left[p_{1} / q_{1}\right]<\left[\bar{p}_{1} / \bar{q}_{1}\right]=\bar{r}_{1}$, then $p_{1}-\left(r_{1}+1\right) q_{1}<0$ and $\bar{p}_{1}-\left(r_{1}+1\right) \bar{q}_{1} \geq 0$. If $\bar{p}_{1}-\left(r_{1}+1\right) \bar{q}_{1} \neq 0$, then $g / h^{r_{1}+1}$ is a $G$-invariant germ of pure meromorphic function, otherwise $g / h^{r_{1}+1}=1 / f^{\left(r_{1}+1\right) q_{1}-p_{1}}$ and $g \cdot\left(g / h^{r_{1}+1}\right)^{p_{1}}$ is a $G$-invariant germ of pure meromorphic function. Arguing inductively along the Euclid's sequences of $(p, q)$ and $(\bar{p}, \bar{q})$ one can always construct a $G$-invariant pure meromorphic function unless $r_{j}=\bar{r}_{j}$ for all $j=1, \cdots, n+1$. But this means that $(p, q)=\left(\alpha s_{n}, \beta s_{n}\right)$ and $(\bar{p}, \bar{q})=\left(\alpha \bar{s}_{n}, \beta \bar{s}_{n}\right)$ for some $\alpha, \beta \in \mathbb{Z}_{+}$. Therefore, $g$, $h$, and $f$ are powers of the same holomorphic function $f^{s_{n}}(\bar{f})^{\bar{s}_{n}}$, thus $f$ and $g$ cannot be generically transverse. A contradiction! The reasoning in the case of many irreducible factors is analogous, being in fact a consequence of the above reasoning. 


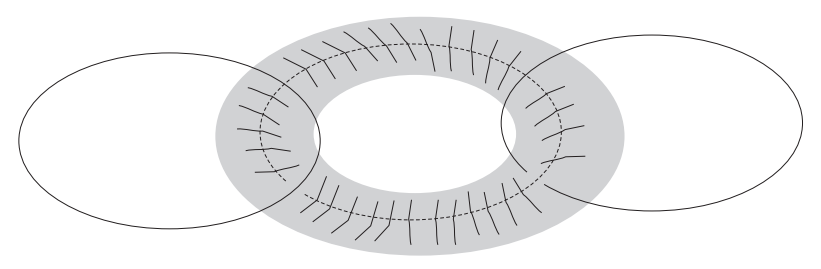

Figure 2 - A dicritical component of $\widetilde{\mathcal{F}}$.

In particular, Theorem 2.3 ensures that the map $G$ defined in Example 2.1 does not preserve the level sets of a couple of generically transverse functions $f, g \in \mathcal{O}_{2}$.

\section{4 - INVARIANT FOLIATIONS}

Let $\omega$ be a germ of holomorphic 1 -form at $0 \in \mathbb{C}^{2}$. Assume that $\operatorname{Sing}(\omega)=\{0\}$ and let $\mathcal{F}_{\omega}$ be the germ of foliation at $0 \in \mathbb{C}^{2}$ given by the Pfaff equation $\omega=0$. We denote by $\operatorname{Aut}\left(\mathcal{F}_{\omega}\right)$ the subgroup of $\operatorname{Diff}\left(\mathbb{C}^{2}, 0\right)$ given by those $\phi \in \operatorname{Diff}\left(\mathbb{C}^{2}, 0\right)$ preserving $\mathcal{F}_{\omega}$, i.e., such that $\phi^{*} \omega \wedge \omega=0$.

Given a map $\phi \in \operatorname{Diff}_{1}\left(\mathbb{C}^{2}, 0\right)$, we have $\phi(x, y)=\exp [1] \widehat{X}(x, y)$ for a (unique) formal vector field $\widehat{X}$ of order at least two (cf. Brochero Martínez et al. 2008, Câmara and Scárdua 2012) called the infinitesimal generator of $\phi$. Let $\mathcal{F}$ be a germ of foliation at $0 \in \mathbb{C}^{2}$ having a dicritical component (i.e., admitting infinitely many separatrices through the origin). We shall say that $\mathcal{F}$ is adapted to $\phi$ if there is a resolution $\pi:(\mathcal{M}, D) \longrightarrow\left(\mathbb{C}^{2}, 0\right)$ of $\widehat{X}$ such that $\pi^{*}(\mathcal{F})$ has infinitely many curves transverse to $D$ (this happens precisely when we blow-up a dicritical component of $\mathcal{F}$ along the resolution of $\widehat{X}$ ).

Lemma 2.4. Let $\mathcal{F}_{\omega}$ be a germ of foliation at $\left(\mathbb{C}^{2}, 0\right)$ having a dicritical component and adapted to $\phi \in$ $\operatorname{Diff}_{1}\left(\mathbb{C}^{2}, 0\right)$. Then $\phi \in \operatorname{Aut}\left(\mathcal{F}_{\omega}\right)$ having finite orbits if and only if $\phi$ is the identity.

Proof. For simplicity we shall write $\mathcal{F}=\mathcal{F}_{\omega}$. Let $\pi:(\mathcal{M}, D) \rightarrow\left(\mathbb{C}^{2}, 0\right)$ be the resolution of $\phi$ introduced in Abate M. 2001, $\widetilde{\mathcal{F}}:=\pi^{*} \mathcal{F}$ the strict transform of $\mathcal{F}$ via $\pi$, and $\widetilde{\phi}$ the lifting of $\phi$. Since $\phi \in \operatorname{Diff}_{1}\left(\mathbb{C}^{2}, 0\right)$, then $\left.\widetilde{\phi}\right|_{D}=\left.\operatorname{Id}\right|_{D}$. If $\widetilde{\mathcal{F}}_{j} \subset \widetilde{\mathcal{F}}$ is a dicritical component of $\widetilde{\mathcal{F}}$ defined in a neighborhood of the irreducible component $D_{j} \subset D$, then it is given in appropriate coordinate systems by a fibration transversal to $D_{j}$, up to a finite number of singular leaves or smooth leaves tangent to $D_{j}$. More precisely, there is an open set $U_{j}:=D_{j} \backslash\left\{p_{1}, \cdots, p_{r}\right\}$ such that $\left.\widetilde{\phi}\right|_{U_{j}}$ can be seen as a family of germs of automorphisms of $(\mathbb{C}, 0)$ with parameters in $U_{j} \subset D_{j} \simeq \mathbb{C P}^{1}$ (see Figure 1). Let $\widetilde{\phi}_{t} \in(\mathbb{C}, 0)$ be given by $\widetilde{\phi}_{t}(x):=\widetilde{\phi}(t, x)$ for some $t \in U_{j}$, then the classical Leau-Fatou flower theorem (Bracci 2004) says that $\widetilde{\phi}_{t}$ has a parabolic fixed point at the origin, unless it is the identity. The result then follows by analytic continuation.

\section{5 - STABILITY}

A germ of map $G \in \operatorname{Diff}\left(\mathbb{C}^{2}, 0\right)$ is said to be positively semistable if for any representative $G: U \longrightarrow G(U)$, where $U$ is an open neighborhood of the origin, and any open set $V \subset U$ there is an open subset $W \subset V$ such that $G^{\circ(n)}(W) \subset V$ for all $n \in \mathbb{Z}_{+}$, i.e., all positive iterates of $G$ starting in $W$ remain in $V$. Notice that any hyperbolic attractor satisfy the previous condition, but if we add the hypothesis of finiteness of the positive orbits, then the map must be periodic. 
Lemma 2.5. Let $G \in \operatorname{Diff}\left(\mathbb{C}^{2}, 0\right)$ be represented by the map $G: U \longrightarrow V$, where $U, V \subset \mathbb{C}^{2}$ are open neighborhoods of the origin with compact closure. Suppose that:

1. $G$ is positively semistable.

2. G has finite orbits.

Then $G$ is periodic, i.e., there is $p \in \mathbb{Z}_{+}$such that $G^{\circ p}=\mathrm{Id}$.

Proof. By the positive semistability, there are $W$ and $V$ as above with $W \subset V$ and satisfying $G^{\circ(n)}(x) \subset V$ for all $x \in W$ and $n \in \mathbb{Z}_{+}$. Now consider the analytic set $C_{q}:=\left\{x \in W: G^{\circ q}=x\right\}$, where $q \in \mathbb{Z}_{+}$. Then $C_{q}$ is a closed set without interior points. Since the orbits of $G$ are all periodic we have $W=\bigcup_{q=1}^{\infty} C_{q}$. From Baire's category theorem, some $C_{q}$ must have an interior point and therefore by the Identity Principle $G$ is periodic.

\section{3 - APPLICATIONS TO FOLIATIONS}

\section{1 - INTEGRABILITY, FIRST INTEGRALS, AND CLOSED ORBITS}

The problem of deciding whether a vector field or, more generally, an ordinary differential equation can be integrated by studying its number of non-transcendent solutions goes back to H. Poincaré, Dulac (cf. Dulac 1912) and other authors. The classical theorem of G. Darboux (cf. Jouanolou 1979) states that a polynomial vector field in the complex plane admits a rational first integral provided that it admits infinitely many algebraic solutions. Our natural framework is the class of analytic equations. With the arrival of the Theory of Foliations the use of geometrical/topological methods has given an important contribution to the comprehension of the problem as well as some important results. Indeed, a holomorphic vector field $X$ defined in a neighborhood $U \subset \mathbb{C}^{n}, n \geq 2$, of the origin $0 \in \mathbb{C}^{n}$, with an isolated singularity at the origin, defines a germ of one-dimensional holomorphic foliation (with a singularity at the origin), and vice-versa.

In dimension two (or codimension one) a classical result (cf. Mattei and Moussu 1980) states that a germ of holomorphic vector field at the origin of $\mathbb{C}^{2}$ admits a holomorphic first integral if and only if : (i) the leaves are closed off the origin; (ii) only finitely many of them are separatrices, i.e., adhere to the origin.

Condition (ii) is usually known as non-dicriticalness of the (germ of) foliation induced by the (germ of) vector field (cf. Camacho and Sad 1982). A foliation germ admitting a pure meromorphic first integral is necessarily dicritical. An example of Suzuki shows that there is no such topological criteria for existence of a meromorphic first integral (cf. Suzuki 1977, Klughertz 1992). Also interesting is the point of view adopted in Alexander and Verjovsky 1988, where the authors prove the existence of a holomorphic first integral for a germ of singular holomorphic vector field in dimension $n \geq 2$, under the hypothesis of existence of a uniform bound for the volume of the orbits of the vector field and some additional condition that restricts the "dicritical case".

Our goal is to investigate topological conditions assuring the existence of holomorphic first integrals for vector field germs in dimension 3. This is done in Theorem 3.4. In few words, our result shows, for a generic class of singularities, an equivalence between the existence of a holomorphic first integral and the fact that the orbits are closed off the origin, plus the existence of a suitable stable separatrix, or the existence of a suitable flag, i.e., a codimension one foliation containing the orbits of the vector field. Our result may be seen as a kind of Reeb stability theorem for singularities of complex vector fields. 
Let us introduce the notation we use, already used in Câmara and Scárdua 2009. Denote the ring of germs of holomorphic functions at $\left(\mathbb{C}^{n}, 0\right)$ by $\mathcal{O}_{n}$ and its maximal ideal by $\mathcal{M}_{n}$. By $\mathfrak{X}\left(\mathbb{C}^{n}, 0\right)$ we denote the $\mathcal{O}_{n}$-module of germs at the origin $0 \in \mathbb{C}^{n}$ of holomorphic vector fields. Given a germ $X \in \mathfrak{X}\left(\mathbb{C}^{n}, 0\right)$, we denote by $\mathfrak{F}_{X}$ the germ of one-dimensional holomorphic foliation at $\left(\mathbb{C}^{n}, 0\right)$ induced by $X$. Let us make clear the notions we use:

Definition 3.1 (holomorphic first integral). Let $\mathfrak{F}_{X}$ be a germ of one-dimensional holomorphic foliation at $0 \in \mathbb{C}^{n}, n \geq 2$. A germ of holomorphic map $F:\left(\mathbb{C}^{n}, 0\right) \rightarrow\left(\mathbb{C}^{n-1}, 0\right)$ is a holomorphic first integral for $\mathfrak{F}_{X}$ if:

(a) $F$ is a submersion off a codimension $\geq 1$ analytic subset.

(b) The leaves of $\mathfrak{F}_{X}$ are contained in the level curves of $F$.

A meromorphic function germ $f \in \mathcal{M}_{n}$ at $0 \in \mathbb{C}^{n}$ is called $\mathfrak{F}_{X}$-invariant if the leaves of $\mathfrak{F}_{X}$ are contained in the level sets of $f$. This can be written as $i_{X}(d f)=X(f) \equiv 0$.

\section{2 - NON-DEGENERATE GENERIC VECTOR FIELD GERMS}

Next we describe the class of vector field germs we shall work with. A germ $X \in \mathfrak{X}\left(\mathbb{C}^{n}, 0\right)$ is non-degenerate if its linear part $D X(0) \in \operatorname{Lin}\left(\mathbb{C}^{n}\right)$ is non-singular. Generically (in terms of the Krull topology for the coefficients of $X)$ the map $D X(0) \in \operatorname{Lin}\left(\mathbb{C}^{n}\right)$ has $n$ distinct eigenvalues, is diagonalizable, and $X$ has an isolated singularity at the origin. From Poincaré-Dulac, Siegel, and Brjuno linearization theorems and from Camacho, Kuiper, and Palis 1978, generically (i.e., for a full measure subset of the set of the set of germs of holomorphic vector fields), up to a change of coordinates, the vector field $X$ leaves invariant the coordinate hyperplanes $x_{1} \cdots x_{n}=0$. We then introduce the following definition:

Definition 3.2 (Non-degenerate generic vector field germs). We shall say that $X$ is non-degenerate generic if $D X(0)$ is non-singular, diagonalizable and, after some suitable change of coordinates, $X$ leaves invariant the coordinate planes.

We shall denote by $\operatorname{Gen}\left(\mathfrak{X}\left(\mathbb{C}^{n}, 0\right)\right)$ the set of germs of non-degenerate generic vector fields at $\left(\mathbb{C}^{n}, 0\right)$. Let $X \in \operatorname{Gen}\left(\mathfrak{X}\left(\mathbb{C}^{n}, 0\right)\right)$ and $S$ be a separatrix of $X$, i.e., an analytic curve germ invariant by $\mathfrak{F}_{X}$ intersecting the origin. From the Newton-Puiseux parametrization theorem one knows that $S \backslash\{0\}$ has the topology of a punctured disc $\mathbb{D} \backslash\{0\}$. Then we denote by $\operatorname{Hol}\left(\mathfrak{F}_{X}, S, \Sigma\right)$ the cyclic holonomy of $\mathfrak{F}_{X}$ with respect to $S$ evaluated at a section $\Sigma$ transverse to $S$, where $\Sigma \cap S=\left\{q_{\Sigma}\right\}$ is a single point. We can choose $\Sigma$ to be biholomorphic to a disc in $\mathbb{C}^{n-1}$ with center corresponding to $q_{\Sigma}$. With this identification the group $\operatorname{Hol}\left(\mathfrak{F}_{X}, S, \Sigma\right)$ is analytically conjugate to a subgroup of the group $\operatorname{Diff}\left(\mathbb{C}^{n-1}, 0\right)$.

An $\mathfrak{F}_{X}$-invariant germ $f \in \mathcal{M}_{n}$ is called adapted to $\left(\mathfrak{F}_{X}, S\right)$ if it can be written locally in the form $f=g / h$, where $g, h \in \mathcal{O}_{n}$ are relatively prime, $S \subset Z(g) \cap Z(h)$, where $Z(g)$ and $Z(h)$ denote the zero sets of $g$ and $h$ respectively, and $\left.f\right|_{\Sigma}$ is pure meromorphic for a generic transverse section $\Sigma$ to $S$. Given $X, Y \in \operatorname{Gen}\left(\mathfrak{X}\left(\mathbb{C}^{3}, 0\right)\right)$ we have $\mathfrak{F}_{X}=\mathfrak{F}_{Y}$ if and only if for some nonvanishing holomorphic function germ $u$ we have $Y=u X$. In this case we say that $X$ and $Y$ are tangent. Any vector field germ $X \in \operatorname{Gen}\left(\mathfrak{X}\left(\mathbb{C}^{3}, 0\right)\right)$ admitting a holomorphic first integral must satisfy the following condition (cf. Câmara and Scárdua 2009). 
Definition 3.3 (condition $(\star)$ ). A germ of generic vector field $X \in \operatorname{Gen}\left(\mathfrak{X}\left(\mathbb{C}^{3}, 0\right)\right)$ satisfies condition $(\star)$ if there is a real line $L \subset \mathbb{C}$ through the origin containing all the eigenvalues of $X$ and such that not all the eigenvalues belong to the same connected component of $L \backslash\{0\}$.

There is therefore one isolated eigenvalue of $X$. The above condition holds for $X$ if and only if holds for any vector field $Y$ such that $X$ and $Y$ are tangent. Condition $(\star)$ implies that $X$ is in the Siegel domain, but is stronger than this last. Denote by $\lambda(X)$ the isolated eigenvalue of $X$ and by $S_{X}$ its corresponding invariant manifold (the existence is granted by the classical invariant manifold theorem). We call $S_{X}$ the distinguished axis of $X$. We shall say that $X$ is transversely stable with respect to $S_{X}$ if for any representative $X_{U}$ of the germ $X$, defined in an open neighborhood $U$ of the origin, any open section $\Sigma \subset U$ transverse to $S_{X}$ with $\Sigma \cap S_{X}=\left\{q_{\Sigma}\right\}$, and any open set $q_{\Sigma} \in V \subset \Sigma$ there is an open subset $q_{\Sigma} \in W \subset V$ such that all orbits of $X_{U}$ through $W$ intersect $\Sigma$ only in $V$.

In this paper we prove various equivalent conditions for the integrability of a generic germ of complex vector field singularity in dimension three (cf. Theorem 3.4). This full statement involves the notion of flag (Corrêa and Soares 2013, Mol 2011) and Kupka singularities (Calvo-Andrade 1994, Kupka 1964), to be developed later in this paper. For the moment we state the following topological criteria.

Theorem 3.1. For any $X \in \operatorname{Gen}\left(\mathfrak{X}\left(\mathbb{C}^{3}, 0\right)\right)$ the following conditions are equivalent:

1. $\mathfrak{F}_{X}$ has a holomorphic first integral.

2. X satisfies condition $(\star)$ and the leaves of $\mathfrak{F}_{X}$ are closed off the origin and transversely stable with respect to $S_{X}$.

From this result we conclude the invariance of the existence of a holomorphic first integral for generic germs in dimension three under topological equivalence.

Corollary 3.2. Let $X, Y \in \operatorname{Gen}\left(\mathfrak{X}\left(\mathbb{C}^{3}, 0\right)\right)$ be generic germs of holomorphic vector fields, both satisfying condition $(\star)$. Assume that $X$ and $Y$ are topologically equivalent. Then $X$ has a holomorphic first integral if and only if $Y$ admits a holomorphic first integral.

We stress that Theorem 3.1 above can be completed (cf. Theorem 3.4) by weakening the topological hypothesis on the orbits, replacing the transverse stability by the existence of a suitable flag, i.e., a codimension one foliation tangent to $\mathfrak{F}_{X}$.

\section{3 - CLOSED LEAVES AND FIRST INTEGRALS}

We show that the closing of the leaves is not sufficient to ensure the existence of first integrals for $\mathfrak{F}_{X}$ with $X \in \operatorname{Gen}\left(\mathfrak{X}\left(\mathbb{C}^{3}, 0\right)\right)$. We first remark (cf. also Câmara and Scárdua 2009, Proposition 1, Section 2.3) that for a generic vector field germ $X$ the local holonomy group $\operatorname{Hol}\left(\mathfrak{F}_{X}, S_{X}, \Sigma\right)$ is generated by a resonant map preserving two smooth curves crossing transversely. In particular, one cannot expect a map like the map $G$ in Example 2.1 appearing as the (generator of the) holonomy of some $X \in \operatorname{Gen}\left(\mathfrak{X}\left(\mathbb{C}^{3}, 0\right)\right)$ with respect to $S_{X}$. Thus, we blow up such map and look to a neighborhood of the point determined by the exceptional divisor and the strict transform of $(y=0)$. Let $X \in \mathfrak{X}\left(\mathbb{C}^{3}, 0\right)$ be given by

$$
X(x)=-m_{1}\left[x_{1}\left(1+a_{1}(x)\right)+x_{2} b_{1}(x)\right] \frac{\partial}{\partial x_{1}}-m_{2} x_{2}\left(1+a_{2}(x)\right) \frac{\partial}{\partial x_{2}}+x_{3} \frac{\partial}{\partial x_{3}}
$$


where $m_{1}, m_{2} \in \mathbb{Z}_{+}, S:=\left(x_{1}=x_{2}=0\right)$, and $\Sigma:=\left(x_{3}=1\right)$. Now consider the closed loop $\gamma$ : $[0,1] \longrightarrow S$ given by $\gamma(t)=\left(0,0, e^{2 \pi \mathbf{i} t}\right)$ and let $\bar{\Gamma}_{\left(x_{1}, x_{2}\right)}(t)=\left(\Gamma_{1}\left(t, x_{1}, x_{2}\right), \Gamma_{2}\left(t, x_{1}, x_{2}\right), \gamma(t)\right)$ be its lifting along the leaves of $\mathfrak{F}_{X}$ starting at $\left(x_{1}, x_{2}, 1\right) \in \Sigma$. In particular, the map $h \in \operatorname{Diff}\left(\mathbb{C}^{2}, 0\right)$ given by $\bar{\Gamma}_{\left(x_{1}, x_{2}\right)}(1)=\left(h\left(x_{1}, x_{2}\right), 1\right)$ is a generator of $\operatorname{Hol}\left(\mathfrak{F}_{X}, S, \Sigma\right)$. Since $\bar{\Gamma}_{\left(x_{1}, x_{2}\right)}(t)$ belongs to a leaf of $\mathfrak{F}_{X}$, then

$$
\frac{\partial}{\partial t} \bar{\Gamma}_{\left(x_{1}, x_{2}\right)}(t)=\alpha X\left(\Gamma_{1}\left(t, x_{1}, x_{2}\right), \Gamma_{2}\left(t, x_{1}, x_{2}\right), \gamma(t)\right)
$$

From this vector equation one has $\gamma^{\prime}(t)=\alpha \gamma(t)$, thus $\alpha=2 \pi \mathbf{i}$. Furthermore

$$
\begin{aligned}
& \frac{\partial}{\partial t} \Gamma_{1}=-2 m_{1} \pi \mathbf{i}\left[\Gamma_{1} \cdot\left(1+a_{1}\left(\Gamma_{1}, \Gamma_{2}, \gamma\right)\right)+\Gamma_{2} \cdot b_{1}\left(\Gamma_{1}, \Gamma_{2}, \gamma\right)\right] \\
& \frac{\partial}{\partial t} \Gamma_{2}=-2 m_{2} \pi \mathbf{i} \Gamma_{2} \cdot\left(1+a_{2}\left(\Gamma_{1}, \Gamma_{2}, \gamma\right)\right) .
\end{aligned}
$$

Example 3.1. Let $X(x)=-\left[x_{1}+x_{2}^{2} b\left(x_{3}\right)\right] \frac{\partial}{\partial x_{1}}-3 x_{2} \frac{\partial}{\partial x_{2}}+x_{3} \frac{\partial}{\partial x_{3}}$, then $S:=\left\{x_{1}=x_{2}=0\right\}$ is invariant by $X$ and the holonomy of $\mathfrak{F}_{X}$ with respect to $S$ evaluated at $\Sigma=\left(x_{3}=1\right)$ has the form $h=\left(h_{1}, h_{2}\right)$ with $h_{j}\left(x_{1}, x_{2}\right)=\Gamma_{j}\left(1, x_{1}, x_{2}\right)$, where $\Gamma_{1}$ and $\Gamma_{2}$ satisfy respectively equations (1) and (2) above. Now if we let $\Gamma_{n}\left(t, x_{1}, x_{2}\right)=\sum_{i+j \geq 1} c_{i, j}^{n}(t) x_{1}^{i} x_{2}^{j}$, then (2) is written in the form

$$
\frac{\partial}{\partial t} \Gamma_{2}=-6 \pi \mathbf{i} \Gamma_{2}
$$

More precisely $\frac{d}{d t} c_{i, j}^{2}(t)=-6 \pi \mathbf{i} \cdot c_{i, j}^{2}(t)$, thus $c_{i, j}^{2}(t)=\lambda_{i, j}^{2} \exp (-6 \pi \mathbf{i} t)$ for some $\lambda_{i, j}^{2} \in C$. Since $\Gamma_{2}\left(0, x_{1}, x_{2}\right)=x_{2}$, then $\lambda_{0,1}^{2}=1$ and $\lambda_{i, j}^{2}=0$ otherwise. Therefore $\Gamma_{2}\left(t, x_{1}, x_{2}\right)=\exp (-6 \pi i t) \cdot x_{2}$ and $h_{2}\left(x_{1}, x_{2}\right)=x_{2}$. On the other hand, (1) is written in the form

$$
\frac{\partial}{\partial t} \Gamma_{1}=-2 \pi \mathbf{i}\left[\Gamma_{1}+e^{-6 \pi \mathbf{i} t} x_{2}^{2} b(\gamma(t))\right]=-2 \pi \mathbf{i}\left(\Gamma_{1}+e^{-6 \pi \mathbf{i} t} b\left(e^{2 \pi \mathbf{i} t}\right) \cdot x_{2}^{2}\right) .
$$

Analogously, $\frac{d}{d t} c_{i, j}^{1}(t)=-2 \pi \mathbf{i} \cdot c_{i, j}^{1}(t)$ for all $(i, j) \neq(0,2)$. Since $\Gamma_{1}\left(0, x_{1}, x_{2}\right)=x_{1}$, then $c_{1,0}^{1}(t)=$ $\exp (-2 \pi i t) \cdot x_{1}$ and $c_{i, j}^{1}(t)=0$ for all $(i, j) \notin\{(1,0),(0,2)\}$. Finally $\frac{d}{d t} c_{0,2}^{1}(t)=-2 \pi i\left(c_{0,2}^{1}(t)+\right.$ $\left.e^{-6 \pi \mathbf{i} t} b\left(e^{2 \pi \mathbf{i} t}\right)\right)$. Now recall that the solution to the Cauchy problem

$$
\alpha^{\prime}(t)=-2 \pi \mathbf{i} \cdot \alpha(t)-2 \pi \mathbf{i} e^{-6 \pi \mathbf{i} t} b\left(e^{2 \pi \mathbf{i} t}\right), \alpha(0)=0
$$

is given by

$$
\alpha(t)=-2 \pi \mathbf{i} e^{-2 \pi \mathbf{i} t} \int_{0}^{t} e^{2 \pi \mathbf{i} s} e^{-6 \pi \mathbf{i} s} b\left(e^{2 \pi \mathbf{i} s}\right) d s=-e^{-2 \pi \mathbf{i} t} \int_{0}^{t} e^{-6 \pi \mathbf{i} s} b\left(e^{2 \pi \mathbf{i} s}\right) 2 \pi i e^{2 \pi \mathbf{i} s} d s
$$

In particular, $\alpha(1)=-e^{-2 \pi \mathbf{i}} \int_{\gamma} \frac{b(z)}{z^{3}} d z$. Thus, if we set $b(z)=-z^{2} / 2 \pi \mathbf{i}$, then $\alpha(1)=1$ and $h\left(x_{1}, x_{2}\right)=$ $\left(x_{1}+x_{2}^{2}, x_{2}\right)$.

Completing the above example we obtain:

Proposition 3.3. There is a vector field $X \in \operatorname{Gen}\left(\mathfrak{X}\left(\mathbb{C}^{3}, 0\right)\right)$ which satisfies condition $(\star)$ and has all leaves closed but does not admit a holomorphic first integral. 
Proof. We consider the vector field $X(x, y, z)=-\left[x-\frac{1}{2 \pi \mathbf{i}} y^{2} z^{2}\right] \frac{\partial}{\partial x}-3 y \frac{\partial}{\partial y}+z \frac{\partial}{\partial z}$. Then $X \in$ $\operatorname{Gen}\left(\mathfrak{X}\left(\mathbb{C}^{3}, 0\right)\right)$. After one blow up along the $z$-axis one has

$$
\begin{aligned}
\pi^{*} X(t, x, z) & =-\left(x-\frac{1}{2 \pi \mathbf{i}} t^{2} x^{2} z^{2}\right) \frac{\partial}{\partial x}+\frac{1}{x}\left(-3 t x-t\left(-x+t^{2} x^{2} z^{2}\right)\right) \frac{\partial}{\partial t}+z \frac{\partial}{\partial z} \\
& =-x\left(1-\frac{1}{2 \pi \mathbf{i}} t^{2} x z^{2}\right) \frac{\partial}{\partial x}-t\left(2+t^{2} x z^{2}\right) \frac{\partial}{\partial t}+z \frac{\partial}{\partial z}
\end{aligned}
$$

which has an isolated singularity at the origin, and whose holonomy with respect to the $z$-axis is precisely the map $\widetilde{G}$ in Example 2.1. Thus it satisfies condition $(\star)$ and has all leaves closed but, from Proposition 2.3 and from what we have observed in Example 3.1 above, $\mathfrak{F}_{X}$ does not admit a holomorphic first integral.

\section{4 - STABILITY, FLAGS, AND FIRST INTEGRALS}

Proposition 3.3 shows that the statements of Theorems 1.2 and 1.3 in Câmara and Scárdua 2009 are incomplete. The correct statement involves a natural adaptation of a classical notion for regular smooth foliations.

\subsection{1 - Stability}

We consider a germ $X \in \operatorname{Gen}\left(\mathfrak{X}\left(\mathbb{C}^{3}, 0\right)\right)$ satisfying condition $(\star)$.

Definition 3.4 (stability). The germ $X$ is transversely stable with respect to $S_{X}$ if for any representative $X_{U}$ of the germ $X$, defined in an open neighborhood $U$ of the origin, any open section $\Sigma \subset U$ transverse to $S_{X}$ with $\Sigma \cap S_{X}=\left\{q_{\Sigma}\right\} \neq\{0\}$, and any open set $q_{\Sigma} \in V \subset \Sigma$ there is an open subset $q_{\Sigma} \in W \subset V$ such that all orbits of $X_{U}$ through $W$ intersect $\Sigma$ only in $V$.

As above mentioned, Definition 3.4 is a natural adaptation to our singular framework of the classical notion of stability due to Lyapunov (Lyapunov 1892) and rediscovered by Reeb (cf. Godbillon 1991).

\subsection{2 - Flags, dicritical components, and Kupka singularities}

Let us first recall some basic notions from singularities of foliations in dimension two. Let $\mathcal{F}$ be a germ of singular foliation at the origin $0 \in \mathbb{C}^{2}$, then Seidenberg's theorem (Seidenberg 1968) gives a reduction of the singularities of $\mathcal{F}$ by the blow-up method. This is also called desingularization or resolution of $\mathcal{F}$. We say that $\mathcal{F}$ has a dicritical component if its resolution contains a non-invariant projective line. This is equivalent to say that $\mathcal{F}$ has infinitely many separatrices, i.e., infinitely many analytic leaves intersecting the origin (Camacho and Sad 1982).

Given $X \in \mathfrak{X}\left(\mathbb{C}^{3}, 0\right)$, we denote its corresponding foliation by $\mathfrak{F}_{X}$. By a flag containing $\mathfrak{F}_{X}$, we mean a germ of codimension one holomorphic foliation $\mathfrak{F}_{\omega}$ at $0 \in \mathbb{C}^{3}$ giving by an integrable holomorphic 1 -form $\omega=A d x+B d y+C d z$ with singular set $\operatorname{Sing}\left(\mathfrak{F}_{\omega}\right)$ of codimension $\geq 2$ containing the origin and with the property that (for some representatives of each foliation defined in a common domain containing the origin) each leaf of $\mathfrak{F}_{X}$ is contained in some leaf of $\mathfrak{F}_{\omega}$. This last property is enclosed in the formula $i_{X} \omega \equiv 0$. The notion of flag is detailed in Mol 2011. A codimension two irreducible component $K \subset \operatorname{Sing}\left(\mathfrak{F}_{\omega}\right) \backslash\{0\}$ is a Kupka type component if $d \omega$ does not vanish along $K$. According to Kupka's theorem (Calvo-Andrade 1994, Kupka 1964), for a representative $\mathfrak{F}_{U}$ of $\mathfrak{F}_{\omega}$ in an open neighborhood $0 \in U$, where $\mathfrak{F}_{\omega}$ is given by an 
integrable holomorphic 1-form $\omega_{U}$, and a representative $K_{U} \subset \operatorname{Sing}\left(\mathfrak{F}_{U}\right)$ of the component $K \subset \operatorname{Sing}\left(\mathfrak{F}_{\omega}\right)$, there is a germ of foliation $\eta(K)$ at $0 \in \mathbb{C}^{2}$ such that for each point $q \in K_{U}$ there is a holomorphic submersion $\varphi_{q}: V_{q} \rightarrow \mathbb{C}^{2}$, with the property that $q \in V_{q} \subset U, \varphi_{q}(q)=0$ and $\varphi_{q}^{*}(\eta(K))=\left.\mathfrak{F}_{U}\right|_{V_{q}}$. The foliation $\eta(K)$ is then called the Kupka transverse type of $\mathfrak{F}_{\omega}$ along the Kupka component $K$. One says that the Kupka component $K$ is dicritical if the corresponding transverse type $\eta(K)$ has a dicritical singularity at the origin $0 \in \mathbb{C}^{2}$, in the above sense.

Example 3.2. A particular case of a dicritical Kupka component is the one induced by the codimension one foliation $\mathfrak{F}_{\omega_{m, n}}$, where $\omega_{m, n}=n y d x-m x d y$ and $m, n \in \mathbb{Z}_{+}$. In fact it represents the product foliation $\mathfrak{F}_{X_{m, n}} \times(\mathbb{C}, 0)$ with $X_{m, n}=m x \frac{\partial}{\partial x}+n y \frac{\partial}{\partial y}$.

We shall call a dicritical Kupka component $\eta(K)$ of radial type if in suitable coordinates $\eta(K)$ is given by a 1 -form $\omega_{m, n}$ as above.

Given a flag $\mathfrak{F}_{\omega}$ containing the foliation $\mathfrak{F}_{X}$, consider its restriction $\left.\mathfrak{F}_{\omega}\right|_{\Sigma}$ to a transverse section $\Sigma \approx$ $\left(\mathbb{C}^{2}, 0\right)$ as above. Since $\Sigma$ is transverse to $\mathfrak{F}_{X}$, it is also transverse to $\mathfrak{F}_{\omega}$ off the singular set $\operatorname{Sing}\left(\mathfrak{F}_{X}\right)$ and therefore one may identify the germ induced by $\mathfrak{F}_{\omega}$ at the point $q_{\Sigma}=\Sigma \cap S(X)$ with the germ of foliation at the origin $0 \in \mathbb{C}^{2}$. Then one says that $\left.\mathfrak{F}_{\omega}\right|_{\Sigma}$ is dicritical if this corresponding germ in dimension two is dicritical.

Definition 3.5 (Adapted flag). Let now $X \in \operatorname{Gen}\left(\mathfrak{X}\left(\mathbb{C}^{3}, 0\right)\right)$ with linear part given by $J^{1}(X)=m x \frac{\partial}{\partial x}+$ $n y \frac{\partial}{\partial y}-k z \frac{\partial}{\partial z}$, then the local holonomy generator $h$ of $\mathfrak{F}_{X}$ with respect to the distinguished axis $z$ is periodic with linear part given by $J^{1}(h)(x, y)=\left(\exp \left(-\frac{2 m \pi i}{k}\right) x, \exp \left(-\frac{2 n \pi i}{k}\right) y\right)$. In particular, $\phi:=h^{\circ(k)}$ is tangent to the identity. Therefore, this map can be written locally in the form $\phi(x, y)=\exp [1] \widehat{X}(x, y)$, where $\widehat{X}$ is its infinitesimal generator. Then one says that $\left(\mathfrak{F}_{X}, \mathfrak{F}_{\omega}\right)$ is an adapted flag if $\mathfrak{F}_{X} \subset \mathfrak{F}_{\omega}$ is a flag such that $\left.\mathfrak{F}_{\omega}\right|_{\Sigma}$ is a germ of foliation having a dicritical component adapted to $\phi=h^{\circ(k)}$.

Notice that the last definitions are of finite determinacy character. Furthermore, if $\left.\mathfrak{F}_{\omega}\right|_{\Sigma}$ is dicritical, then $\left(\mathfrak{F}_{X}, \mathfrak{F}_{\omega}\right)$ is automatically an adapted flag. Using this terminology, one may complete the statements in Câmara and Scárdua 2009 as follows.

Theorem 3.4. Suppose that $X \in \operatorname{Gen}\left(\mathfrak{X}\left(\mathbb{C}^{3}, 0\right)\right)$ satisfies condition $(\star)$ and let $S_{X}$ be the distinguished axis of $X$. Then the following conditions are equivalent:

1. The leaves of $\mathfrak{F}_{X}$ are closed off the origin and transversely stable with respect to $S_{X}$;

2. $\operatorname{Hol}\left(\mathfrak{F}_{X}, S_{X}, \Sigma\right)$ has finite orbits and is (topologically) stable;

3. $\operatorname{Hol}\left(\mathfrak{F}_{X}, S_{X}, \Sigma\right)$ is periodic;

4. $\mathfrak{F}_{X}$ has a holomorphic first integral.

Moreover, in terms offlags of foliations, the above conditions are also equivalent to each of the following conditions:

5. The leaves of $\mathfrak{F}_{X}$ are closed off the origin and there is an adapted flag $\left(\mathfrak{F}_{X}, \mathfrak{F}_{\omega}\right)$;

6. The leaves of $\mathfrak{F}_{X}$ are closed off the origin and there is a flag $\mathfrak{F}_{X} \subset \mathfrak{F}_{\omega}$ such that $\mathfrak{F}_{\omega}$ is a Kupka component of radial type. 
Proof of the first part of Theorem 3.4. It follows immediately from the definition of transverse stability of germs of vector fields and from (topological) stability of maps that (1) implies (2). It comes from Lemma 2.5 that (2) implies (3). Now let us prove that (3) implies (4). Since $X$ satisfies condition $(\star)$ and $\operatorname{Hol}\left(\mathfrak{F}_{X}, S_{X}, \Sigma\right)$ is linearizable, then Elizarov and Ilyashenko 1984 ensures that $\mathfrak{F}_{X}$ is linearizable. Therefore, one may suppose without loss of generality that $X(x)=\lambda x_{1} \frac{\partial}{\partial x_{1}}+\mu x_{2} \frac{\partial}{\partial x_{2}}-\kappa x_{3} \frac{\partial}{\partial x_{3}}$, where $\lambda, \mu, \kappa \in \mathbb{R}_{+}$. Since $\operatorname{Hol}\left(\mathfrak{F}_{X}, S_{X}, \Sigma\right)$ is periodic, one may suppose without loss of generality that $\lambda=m, \mu=n, \kappa=k \in \mathbb{Z}_{+}$. The result then follows from Lemma 2.3 in Câmara and Scárdua 2009. Finally let us verify that (4) implies (1). The existence of a first integral for $\mathfrak{F}_{X}$ ensures that the leaves of $\mathfrak{F}_{X}$ are closed off $\operatorname{Sing}\left(\mathfrak{F}_{X}\right)$. Furthermore, $\operatorname{Hol}\left(\mathfrak{F}_{X}, S_{X}, \Sigma\right)=\langle H\rangle$ admits a couple of generically transverse $\mathfrak{F}_{X}$-invariant holomorphic functions whose restrictions to $\Sigma$ have the level sets preserved by $H$. Thus Theorem 2.3 ensures that $\operatorname{Hol}\left(\mathfrak{F}_{X}, S_{X}, \Sigma\right)$ is periodic and, in particular, topologically stable. Hence the leaves of $\mathfrak{F}_{X}$ are transversely stable with respect to $S_{X}$. This proves the first four equivalences in Theorem 3.4.

As a straightforward consequence (cf. Theorem 2 in Câmara and Scárdua 2009), one has the following topological criterion for the existence of invariant meromorphic functions for elements in $\operatorname{Gen}\left(\mathfrak{X}\left(\mathbb{C}^{3}, 0\right)\right)$.

Theorem 3.5. Let $X \in \operatorname{Gen}\left(\mathfrak{X}\left(\mathbb{C}^{3}, 0\right)\right)$ satisfy condition $(\star)$ with distinguished axis $S_{X}$. Suppose that $\mathfrak{F}_{X}$ has closed leaves off the origin and is transversely stable with respect to $S_{X}$. Then there is an $\mathfrak{F}_{X}$-invariant meromorphic function adapted to $\left(\mathfrak{F}_{X}, S_{X}\right)$.

Now we study the topological invariance of the existence of a holomorphic first integral for a generic germ of holomorphic vector field, as a consequence of our preceding results. We recall that two germs of holomorphic vector fields $X$ and $Y$ at the origin $0 \in \mathbb{C}^{n}$ are topologically equivalent if there is a homeomorphism $\psi: U \rightarrow V$, where $U, V$ are neighborhoods of the origin $0 \in \mathbb{C}^{n}$ and $X_{U}, Y_{V}$ are vector fields representing $X, Y$ respectively, such that $\psi$ takes orbits of $X_{U}$ into orbits of $Y_{V}$. Such a map $\psi$ takes separatrices of $X_{U}$ into separatrices of $Y_{V}$ : indeed, a separatrix of $X_{U}$ is an orbit which is closed off the origin, and the same holds for its image under $\psi$. Assume that the vector field $X$ is generic satisfying condition $(\star)$ and admits a holomorphic first integral. In this case one has:

Claim 6. The vector field $X$ is analytically linearizable, say $X(x, y, z)=X_{n, m,-k}:=n x \frac{\partial}{\partial x}+m y \frac{\partial}{\partial y}-k \frac{\partial}{\partial z}$ with $n, m, k \in \mathbb{Z}_{+}$in suitable local coordinates $(x, y, z) \in\left(\mathbb{C}^{3}, 0\right)$. In particular, $X$ admits a unique separatrix off the dicritical plane $\{z=0\}$, and this separatrix corresponds to the distinguished separatrix $S_{X}$

Proof. Indeed, the analytic linearization of $X$ is a straightforward consequence of the first part of Theorem 3.4 (or, since by hypothesis there is a holomorphic first integral, in view of Lemma 2.5 and Elizarov and Ilyashenko 1984). In this normal form

$$
X(x, y, z)=X_{n, m,-k}:=n x \frac{\partial}{\partial x}+m y \frac{\partial}{\partial y}-k \frac{\partial}{\partial z}
$$

the "dicritical plane" is the plane $\{z=0\}$ and the distinguished separatrix is the $z$-axis. The orbit $\mathcal{O}_{(a, b, c)}$ of $X$ through the point $(a, b, c)$ is given by

$$
\phi(t)=(x(t), y(t), z(t))=\left(a e^{n t}, b y e^{m t}, c e^{-k t}\right), t \in \mathbb{C} .
$$


Thus, if $\mathcal{O}_{(a, b, c)}$ accumulates at the origin, either $c=0$ or $c \neq 0$ and $a=b=0$. For instance, if $c \neq$ $0 \neq a$, then the orbit is contained in the hypersurface $x^{k} z^{n}=a^{k} c^{n} \neq 0$, which does not accumulate at the origin.

Lemma 3.6. A topological equivalence takes the distinguished axis of $X$ into the distinguished axis of $Y$.

Proof. Indeed, as we have seen above, the image $\psi\left(S_{X}\right)$ is some separatrix of $Y$. If this is not the distinguished axis of $Y$, then the distinguished axis of $Y$ is taken by $\psi^{-1}$ into a separatrix other than the distinguished axis of $X$. Therefore, according to Claim $6, \psi^{-1}\left(S_{Y}\right)$ must be a separatrix of the "dicritical" part of $X$, i.e., in the coordinates $(x, y, z)$ above, where $X(x, y, z)=X_{n, m,-k}$, we have $\psi^{-1}\left(S_{Y}\right) \subset\{z=0\}$. Nevertheless, any invariant neighborhood of a leaf contained in a dicritical separatrix of $X$ off the origin intersects infinitely many separatrices (namely, those contained in the intersection of this neighborhood with the dicritical plane $\{z=0\}$ ). On the other hand, this same phenomena does not occur for arbitrarily small invariant neighborhoods of a leaf contained in the distinguished axis $S_{Y}$ of $Y$. Therefore, necessarily $\psi\left(S_{X}\right)$ is the distinguished axes of $Y$.

From the above considerations we immediately obtain Corollary 3.2 from Theorem 3.4.

\subsection{3 - Flags and integrability}

In this section we prove the remaining part of Theorem 3.4. We address therefore the following problem. Given a germ of foliation by curves $\mathfrak{F}_{X}$ induced by a germ of vector field of the form

$$
X=m x(1+a(x, y, z)) \frac{\partial}{\partial x}+n y(1+b(x, y, z)) \frac{\partial}{\partial x}-k z\left(1+c(x, y, z) \frac{\partial}{\partial z}\right.
$$

with $a, b, c \in \mathcal{M}_{3}$, what are the consequences of the existence of a codimension 1 germ of holomorphic foliation tangent to $X$, which is transversely dicritical with respect to $S$ ?

We begin by studying the consequences of the existence of a flag foliation with a dicritical transverse type for a vector filed $X \in \operatorname{Gen}\left(\mathfrak{X}\left(\mathbb{C}^{3}, 0\right)\right)$.

Lemma 3.7. Let $\mathfrak{F}_{X}$ be a germ of foliation by curves at $\left(\mathbb{C}^{3}, 0\right)$, S an invariant curve of $\mathfrak{F}_{X}$ through the origin, and $\mathfrak{F}_{\omega}$ a codimension one foliation satisfying the following conditions:

(i) $\mathfrak{F}_{\omega}$ is tangent to $X$;

(ii) There is a section $\Sigma$ transverse to $S$ such that $\left.\mathfrak{F}_{\omega}\right|_{\Sigma}$ is dicritical.

Then $\mathfrak{F}_{\omega}$ is transversely dicritical with respect to $S$.

Proof. Since the orbits of $X$ are contained in the leaves of $\mathfrak{F}$, then these leaves are invariant by the flow of $X$. Therefore, if $\Sigma^{\prime}$ is another section transversal to $S$ and $h: \Sigma \longrightarrow \Sigma^{\prime}$ is an element of the holonomy pseudogroup of $X$ with respect to $S$, then it is a diffeomorphism taking the leaves of $\left.\mathfrak{F}\right|_{\Sigma}$ onto the leaves of $\left.\mathfrak{F}\right|_{\Sigma^{\prime}}$

We are now in a position to finish the proof of Theorem 3.4. For this sake, let us first recall some facts proved along this work and introduce some terminology. First notice that any $X \in \operatorname{Gen}\left(\mathfrak{X}\left(\mathbb{C}^{3}, 0\right)\right)$ admitting a holomorphic first integral must satisfy condition $(\star)$ in Definition 3.3 (cf. Câmara and Scárdua 


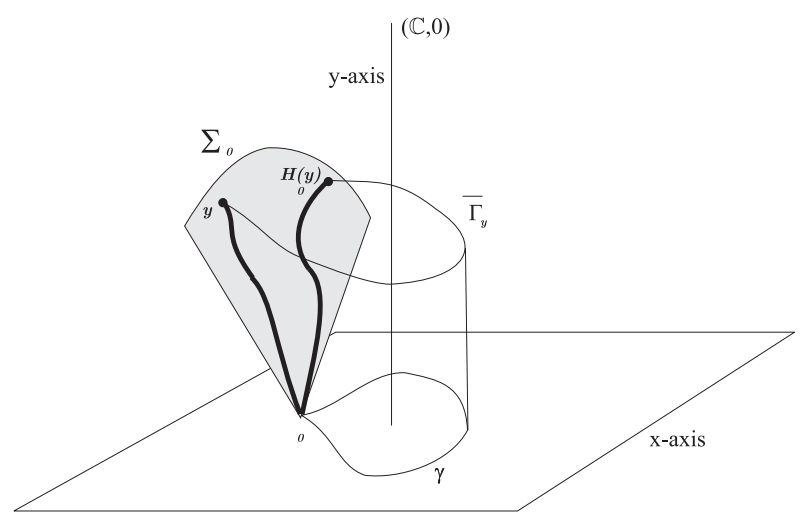

Figure 3 - The liftings of $\gamma$ along the leaves of $\mathfrak{F}_{X}$ starting at points of $L$.

2009). Assume the curve $S_{X}$ is the $z$-axis, let $\Sigma_{z}:=\left(z=\right.$ const.) be a section transverse to $S_{X}$, and $\operatorname{Hol}\left(\mathfrak{F}_{X}, S_{X}, \Sigma_{z}\right)$ be the holonomy of $\mathfrak{F}_{X}$ with respect to $S_{X}$ evaluated at $\Sigma_{z}$.

End of the proof of Theorem 3.4. First suppose all the leaves of $\mathfrak{F}_{X}$ are closed off the origin $\{0\} \subset \mathbb{C}^{3}$ and that there is an adapted flag $\mathfrak{F}_{X} \subset \mathfrak{F}_{\omega}$. Given a germ of leaf $L$ of $\mathfrak{F}_{X}$ it follows that the closure $\bar{L} \subset L \cup\{0\}$ is a germ of analytic subset of pure dimension one (Gunning and Rossi 1965) at $\left(\mathbb{C}^{3}, 0\right)$. Since this leaf is transverse to $\Sigma_{z}$, one concludes that $\bar{L} \cap \Sigma_{z}$ is a finite set. On the other hand, given a point $x \in L \cap \Sigma_{z}$, its orbit in the holonomy group is also contained in $L \cap \Sigma_{z}$, so that it is a finite set. Thus the orbits of the generator of $\operatorname{Hol}\left(\mathfrak{F}_{X}, S_{X}, \Sigma_{z}\right)$ are finite. By hypothesis, for any $z_{0} \in S(X)$ the foliation $\left.\mathfrak{F}_{\omega}\right|_{\Sigma_{z_{0}}}$ has a dicritical component. Now consider a simple loop $\gamma$ around the origin inside the $z$-axis starting from $z_{0}$. Pick a leaf $L$ of $\left.\mathfrak{F}_{\omega}\right|_{\Sigma_{z_{0}}}$ and consider the liftings of $\gamma$ starting at points of $L$, along the trajectories of $\mathfrak{F}_{X}$. Then these liftings form a three dimensional real variety, say $S_{L}$, whose intersection with $\Sigma_{z_{0}}$ is given by $L$ and $L^{\prime}$ (see Figure 2). In particular, if $h:=h_{\gamma}$ is the generator of $\operatorname{Hol}\left(\mathfrak{F}_{X}, S_{X}, \Sigma_{z}\right)$, then $L^{\prime}=h(L)$. For the 1-form $\omega$, one has that $S_{L}$ is tangent to $\operatorname{Ker}(\omega)$ and $S_{L} \cap \Sigma_{z_{0}}$ is tangent to the induced foliation $\mathfrak{F}_{\omega} \mid \Sigma_{z_{0}}$. Thus, $L^{\prime}$ is a leaf of $\left.\mathfrak{F}_{\omega}\right|_{\Sigma_{z_{0}}}$. Since $\left.\mathfrak{F}_{\omega}\right|_{\Sigma_{z_{0}}}$ has a dicritical component and $h$ is a diffeomorphism with resonant linear part having finite orbits, then Lemma 2.4 ensures that $h$ is periodic (in particular linearizable and finite). Since $\mathfrak{F}_{X} \in \operatorname{Gen}\left(\mathfrak{X}\left(\mathbb{C}^{3}, 0\right)\right)$ has linearizable periodic holonomy, then it follows from Elizarov and Ilyashenko 1984 that the foliation $\mathfrak{F}_{X}$ is also analytically linearizable. Therefore, one may suppose without loss of generality that $X(x, y, z)=m x \frac{\partial}{\partial x}+n y \frac{\partial}{\partial y}-k z \frac{\partial}{\partial z}$. This vector field has a holomorphic first integral. From the above linearization, it is easy to see that the flag foliation $\mathfrak{F}_{\omega}$ containing $\mathfrak{F}_{X}$ must have a linear dicritical Kupka transverse type along the $z$-axis. In particular, $\mathfrak{F}_{\omega}$ is of radial type. This proves that (5) implies (1)-(4) and also (6). Since the converse is immediate, this proves that the first four conditions in Theorem 3.4 are equivalent to conditions (5) and (6).

Remark 3.1 (Parabolic curves and smooth sets of fixed points cf. Abate 2001). In our previous paper Câmara and Scárdua 2009 it is stated an integrability result mentioning only the fact that the leaves of $\mathfrak{F}_{X}$ are closed off $\operatorname{Sing}\left(\mathfrak{F}_{X}\right)$. Nevertheless, as we saw above, this result is not correct. Indeed, there are such kind of vector fields without holomorphic first integral (cf. Example 3.3).

Let us identify precisely the missing point in Câmara and Scárdua 2009. This justifies the further topological conditions introduced above in order to correct the statements of the main theorems therein (Theo- 
rems 1.2 and 1.3 in Câmara and Scárdua 2009). Along these lines we shall keep all the notations introduced in Câmara and Scárdua 2009. In Theorem 3.6 of Câmara and Scárdua 2009 we have stated that every non trivial complex map germ fixing the origin admits a parabolic curve. Javier Ribon draw our attention to the fact that this is not true with the following example:

Let $X^{o}=p y \frac{\partial}{\partial y}-q x \frac{\partial}{\partial x}$ with $p, q \in \mathbb{Z}_{+}$and $X=x y X^{o}$, then the orbits of the map $\Phi(x, y)=$ $\exp [1] X(x, y)$ are confined in the level sets of the first integral $f(x, y)=x^{p} y^{q}$ to the vector field $X$. Therefore, $\Phi$ has no orbit attracting to the origin, thus it does not admit any parabolic curve at the origin.

Some time after that Marco Abate communicated us the same fact showing that Theorem 3.6 in Câmara and Scárdua 2009 contradicts Proposition 2.1, p. 185, in Abate 2001. As a matter of fact, Lemma 3.5 (and thus Theorem 3.6) is not correct. This is due to the authors misinterpretation of the proof of Corollary 3.1 in Abate 2001 wrongly stated as Theorem 3.2 in Câmara and Scárdua 2009. Indeed, the correct statement is the following: Let $G \in \operatorname{Diff}_{1}\left(\mathbb{C}^{2}, 0\right)$ and suppose that $S:=\operatorname{Fix}(G)$ is a smooth curve through the origin such that $\operatorname{ind}_{0}(G, S) \notin \mathbb{Q}^{+}$. Then $G$ admits $\nu(f)-1$ parabolic curves.

More precisely, one can check that this would be the appropriate hypothesis looking to the proof of Theorem 3.1 in Abate 2001. Now one can check that the diffeomorphism in the proof of Lemma 3.5 in Câmara and Scárdua 2009 does not satisfy the conditions of the above theorem.

We finish with an immediate consequence of the proof of Theorem 2.3.

Corollary 3.8. Let $X \in \operatorname{Gen}\left(\mathfrak{X}\left(\mathbb{C}^{3}, 0\right)\right)$ and $S_{X}$ be the distinguished axis of $X$. Suppose that $\mathfrak{F}_{X}$ admits a pure meromorphic first integral, then the holonomy group $\operatorname{Hol}\left(\mathfrak{F}_{X}, S_{X}, \Sigma\right)$ is periodic.

\section{4 - REFERENCES}

ABATE M. 2006. The residual index and the dynamics of maps tangent to the identity. Duke Math J 107: 173-207.

ALEXANDER JC AND VERJOVSKY A. 1988. First integrals for singular holomorphic foliations with leaves of bounded volume. Lecture Notes in Math 1345: 1-10.

BRACCI F. 2004. Local dynamics of holomorphic diffeomorphisms. Boll Unione Mat Ital 7-B(8): 609-636.

BROCHERO MARTÍNEZ. 2003. Groups of germs of analytic diffeomorphisms in $\left(\mathbb{C}^{2}, 0\right)$. J Dyn Control Syst 9: 1-32.

BROCHERO MARTÍNEZ, CANO F AND LÓPES-HERNANZ L. 2008. Parabolic curves for diffeomorphisms in $\left(\mathbb{C}^{2}, 0\right)$. Publ Mat 52: 189-194.

CALVO-ANDRADE O. 1994. Irreducible components of the space of holomorphic foliations. Math Ann 299: $751-767$.

CAMACHO C, KUIPER NH AND PALIS J. 1978. The topology of holomorphic flows with singularities. Publ Math Inst Hautes Études Sci 48: 5-38.

CAMACHO C AND SAD P. 1982. Invariant varieties through singularities of holomorphic vector fields. Ann of Math 115(2): 579-595.

CÂMARA LM AND SCÁRDUA BA. 2009. On the integrability of holomorphic vector fields. Discrete Contin Dyn Syst 25: 1-13. CÂMARA LM AND SCÁRDUA BA. 2012. A Fatou type theorem for complex map germs. Conform Geom Dyn 16: $256-268$.

CORREAA JR AND SOARES MG. 2013. Inequalities for characteristic numbers of flags of distributions and foliations. Int J Math 24: 12.

DULAC H. 1912. Solutions d'un système de équations différentiale dans le voisinage des valeus singulières. Bull Soc Math France 40: 324-383.

ELIZAROV PM AND ILYASHENKO YUS. 1984. Remarks on the orbital analytic classification of germs of vector fields. Math USSR-Sb 49: 111-124.

GODBILLON C. 1991. Feuilletages. Études géométriques. With a preface by G. Reeb. Prog in Math 98. Birkh'user Verlag, Basel. GUNNING RC AND ROSSI H. 1965. Analytic functions of several complex variables. Prentice Hall, Englewood Cliffs, NJ.

JOUANOLOU JP. 1979. Équations de Pfaff algébriques. Lecture Notes in Math. 708, Springer-Verlag, Berlin.

KLUGHERTZ M. 1992. Existence d'une intégrale première méromorphe pour des germes de feuilletages à feuilles fermées du plan complexe. Topology 31:255-269. 
KUPKA I. 1964. The singularities of integrable structurally stable Pfaffian forms. Proc Natl Acad Sci USA 52: $1431-1432$.

LYAPUNOV AM. 1892. The General Problem of the Stability of Motion (In Russian). Doctoral dissertation, Univ Kharkov. English translations: (1) Stability of Motion. Academic Press. New-York \& London 1966 (2) The General Problem of the Stability of Motion, (A. T. Fuller trans.) Taylor \& Francis, London 1992.

MATTEI JF AND MOUSSU R. 1980. Holonomie et intégrales premières. Ann Sci Ec Norm Supér 13(4): 469-523.

MOL RS. 2011. Flags of holomorphic foliations. An Acad Bras Cienc 83: 775-786.

SEIDENBERG A. 1968. Reduction of the singularities of the differentiable equation $A d y=B d x$. Amer J Math 90: 248-269.

SUZUKI M. 1977. Sur les intégrales premiéres de certains feuilletages analytiques complexes, in Fonctions de Plusieurs Variables Complexes III, Seminaire François Norguet Octobre 1975, Springer Berlin, Heidelberg, New York, 1978. Lecture Notes in Math 670: 53-79. 